\title{
APLICACIÓN DE LA REFORMULACIÓN ALEMANA DEL MÉTODO DEL TEMA CENTRAL DE CONFLICTO RELACIONAL (CCRT-LU) PARA LA EVALUACIÓN DEL CAMBIO EN UN CASO DE DUELO POR PÉRDIDA DE LA PAREJA
}

\section{APPLICATION OF THE GERMAN REFORMULATION OF THE CORE CONFLICTUAL RELATIONSHIP THEME METHOD (CCRT-LU) TO EVALUATE CHANGES IN A GRIEVING CASE OF THE LOSS OF THE PARTNER}

\author{
Moraima G. García Mantilla \\ Doctora en Fundamentos y Desarrollos Psicoanalíticos, \\ Universidad Complutense de Madrid, España \\ Alejandro Ávila-Espada \\ Catedrático de Psicoterapia. Facultad de Psicología, \\ Universidad Complutense de Madrid.
}

Cómo referenciar este artículo/How to reference this article:

García Mantilla, M.G, y Ávila-Espada, A. (2016). Aplicación de la Reformulación Alemana del Método del Tema Central de Conflicto Relacional (CCRT-LU) para la Evaluación del Cambio en un Caso de Duelo por Pérdida de la Pareja. Revista de Psicoterapia, 27(103), 267-282.

\section{Resumen}

Se aplicó el método CCRT en su reformulación alemana denominada CCRT-LU, utilizando la versión del método en castellano CCRT-LU-S, a un caso único de duelo por pérdida de la pareja. El objetivo fue evaluar clínica y sistemáticamente el cambio en el proceso psicoterapéutico de inicio y fin de la terapia. El método se mostró sensible para identificar el cambio de patrón relacional núcleo de conflicto en el contenido verbal de las sesiones psicoterapéuticas del sujeto, en donde se investigaron los patrones centrales de relación identificables, con el fin de detectar si existe un patrón, guión o esquema típico del duelo. Se trabajó con un modelo de psicoterapia de tiempo limitado y focal desarrollado en 14 sesiones y un seguimiento al mes. El foco de trabajo elegido fue la elaboración del duelo por muerte de la pareja.

Palabras clave: Duelo por muerte de la pareja, Psicoterapia Psicoanalítica Focal, CCRT, CCRT-LU.

\begin{abstract}
The Core Conflictual Relationship Theme Method (CCRT) in its German reformulation named CCRT$L U$, and in the Castilian version named CCRT-LU-S, was applied to a single case of grief for the loss of the partner. The objective was to evaluate clinically and systematicallychanges in the psychotherapeutic process comparing the beginning and the end of the treatment. The method was sensitive to identify in the verbal content of individual psychotherapy sessions the changing patterns of the Core Conflictual Relationship and the central relationship patterns were investigated in order to detect whether there is a pattern, script or outline typical of grief. We worked with focal and brief psychodynamic psychotherapy model of 14 sessions and a follow up after a month. The focus of work in psychotherapy chosen was the elaboration of grieffor death of the spouse.
\end{abstract}

Keywords: Grieving the loss of a partner, Focal Psychoanalytical Psychotherapy, CCRT, CCRT-LU.

Fecha de recepción v1: 06-05/2015. Fecha de recepción v2: 03-06-2015. Fecha de aceptación: 26-02-2016. Correspondencia sobre este artículo: E-mail: moraimamantilla@gmail.com Dirección postal: Caamperstraat 44-3 1091 Amsterdam, Holanda. (C) 2016 Revista de Psicoterapia 


\section{Introducción}

En este trabajo se analizará clínica y sistemáticamente el duelo postmortem de un ser querido (la pareja), cuando se detecta en la práctica clínica que dicho fenómeno cuenta con los elementos necesarios de un proceso de duelo complicado o patológico; ya Freud en 1917, publicó Duelo y Melancolía, en donde describía al duelo como: "La reacción frente a la pérdida de una persona amada o de una abstracción que haga sus veces, como la patria, la libertad, un ideal, etcétera. A raíz de idénticas influencias en muchas personas se observa, en lugar de duelo melancolía y por eso sospechamos en ellas una disposición enfermiza" (p.238).

Siguiendo esta línea de investigación, estudios más recientes plantean que la relación entre proceso de duelo y psicopatología es estrecha: por un lado, el duelo puede girar su rumbo y complicarse al grado de desencadenar desequilibrio mental, y por otro lado, los trastornos psicopatológicos ya establecidos pueden suponer dificultades particulares, en la elaboración del duelo (Bowlby 1980/1993; Brown y Harris 1978; Horowitz y otros 1993; Parkes 1998; Woof y Carter 1997). Pero también existen otros autores que piensan que los duelos ni tienen ni deben tener qué ver con la psiquiatría y sus tratamientos, al menos en primera instancia (Tizón, 2004); dando como resultado que en pleno siglo XXI, la revisión de la literatura muestre posturas heterogéneas y matizadas por el interés particular de cada uno de los investigadores sobre lo que constituye un duelo complicado o patológico.

En esta investigación se tomarán como base las aportaciones que Tizón (2004) ha adoptado sobre los conceptos de duelo complicado y patológico. En donde el autor señala que estos dos tipos de duelo no tienen por qué ser lo mismo aunque frecuentemente se confunde el duelo complicado (ya sea por algunas de las características o variables que inciden en él, o por la evolución que conlleva) con el duelo patológico. En su concepción, el duelo patológico es una forma de duelo complicado, debido a las dificultades que pueden expresarse en el mismo. De igual manera, una buena parte de las manifestaciones en los duelos complicados pueden ser vistas como síntomas (psicopatológicos), pero su evolución posterior y su elaboración las revierten a su primer origen: manifestaciones de duelo complicado.

Este trabajo expone el caso de una paciente que se encuentra elaborando un duelo complicado por la pérdida intempestiva de la pareja. A este tipo de duelo se le conoce en la literatura como duelo de tipo súbito. Dicho proceso de duelo implica en sí una serie de complicaciones por las causas y circunstancias que rodean a la perdida. En este tipo de duelo la muerte se presenta sin previo aviso, por lo que requiere una intervención y comprensión especial debido a que es más difícil de elaborar pues no existe algún aviso anticipado de muerte inminente (Parkes, 1998; Worden, 2004). En este sentido, autores anglosajones propusieron una regla mnemotécnica con respecto a la complicación de los procesos psicológicos del duelo. Se trata del acrónimo NASH (Natural, Accidental, Suicide, Homicide). El cual significa que, cuanto más a la derecha de ese orden se encuentre el desencadenante del duelo concreto, más difícil será elaborar psicológicamente esa pérdida afectiva 
(Tizón \& Sforza, 2008).

Existen otro tipo de factores que ya desde Freud (1917) se han considerado como fundamentales para la elaboración del duelo. Estas variables afectan el curso de los procesos de duelo y entre ellas encontramos las características del objeto perdido (por ej., la pérdida de un ser muy querido), características del sujeto (por ej., personalidades con tendencias a las relaciones ambivalentes); características de la relación entre el sujeto y objeto (por ej., conflictos importantes), de las circunstancias que afectan a la pérdida (por ej., pérdidas inesperadas) entre otras causas (por ej., el sentimiento de haber sido el causante de la pérdida). Sin embargo, estas variables deben tomarse como simples vértices de exploración ya que el proceso de duelo varía de sujeto a sujeto y entre culturas.

Ahora bien, la muerte de la pareja ocupa los primeros lugares en la lista de los provocadores de estrés, y los trastornos depresivos son cuatro veces más frecuentes en las personas que enviudan con respecto a las personas que tienen una pareja (Turvey, Carney, Arndt, Wallace, y Herzog, 1999). Con la vejez resultan más evidentes las manifestaciones del duelo, ya que un duelo complicado puede evolucionar hacia un duelo patológico. Son frecuentes entre las personas mayores que sufren un duelo la aparición de trastornos depresivos y enfermedades cardiacas o circulatorias (Tizón y Sforza, 2008). Por ende, diversos estudios han mostrado tasa alta de mortalidad y morbilidad durante el proceso de duelo por viudez, sobre todo en los ancianos (Parkes, 1998). Todo esto implica la necesidad preventiva de estimular el contacto social y profesional en este tipo de situaciones pues en este tipo de pérdida en particular, muchas viudas o viudos requieren meses o más tiempo para empezar a creer o entender que su pareja ha muerto y no volverá.

Es por ello que, basándonos en la premisa de prevención y fomento del contacto profesional en casos complicados de duelo, en esta investigación nos planteamos trabajar conforme a criterios de investigación con una paciente que enfrentaba un duelo complicado por las variables implicadas. El tratamiento utilizado fue psicoterapia de tiempo limitado, con objetivos específicos y un foco de trabajo claro y asequible en el tratamiento, como se propone en las terapias dinámicas de tiempo limitado, aplicando criterios de la psicoterapia focal (Balint, y otros 1985; Malan 1963). El proceso de tratamiento se encuadró a una intervención de 15 sesiones y un seguimiento al mes. Esta cifra de intervenciones es coherente con el límite que va de 10 a 25 sesiones de varias terapias dinámicas breves (Levenson, 1997). La estrategia de tiempo limitado se hizo presente desde el principio de la intervención, subrayando que la terapia finalizaría en la sesión 14, induciendo mediante la puntuación del tiempo el foco en la terminación, operador especialmente relevante en una intervención sobre duelo.

La intervención psicoterapéutica con la paciente asumió principalmente como objetivo clínico contribuir a la elaboración de duelo; pero la investigación que luego se desarrolló fue independiente de la psicoterapia y se aceptó como hipótesis que el método del Tema Central de Conflicto Relacional (CCRT) desarrollado original- 
mente por Lester Luborsky y Paul Crits Cristoph $(1976,1990,1998)$ en su reformulación alemana denominada CCRT- LU, (Albani, Pokorny, Blazer, Koenig et al., 2002), y en su versión castellana: CCRT-LU-S (López del Hoyo, Ávila Espada, Pokorny, y Albani, 2004), se mostraría sensible en detectar el proceso de duelo y que los logros clínicos se reflejarían igualmente en cambios estructurales en el patrón conflictivo relacional central. Es por ello que el proceso psicoterapéutico de la paciente fue sometido de manera sistemática a la aplicación del método para analizar en el material de las sesiones psicoterapéuticas de la paciente, los patrones centrales de relación que establecen, con el fin de detectar si existe un patrón, guión o esquema (Deseos y Respuestas de los otros y Respuestas del sujeto) típico del duelo.

Existe una amplia variedad de trabajos realizados con el CCRT (véase Luborsky et al., 1999), pero hasta ahora hay pocos trabajos que utilicen este método para describir procesos terapéuticos. Se conocen estudios que hacen referencia a terapias breves (Albani et al., 2002;Anstadt, Merten, Ulrich y Krause, 1996; Grahbotrtn, Overbeck, Kernhof, Jordan y Muller, 1994; Luborsky, Crits-Cristoph, Friedman, Mark y Schaffler, 1991). Pero son mínimos los estudios que describen el proceso terapéutico de un tratamiento psicoanalítico prolongado (Albani et al., 2002). Esta investigación pretende analizar sistemáticamente el proceso en un encuadre de psicoterapia breve de corte psicoanalítico, utilizando la variación alemana del método y este artículo se limita a contribuir a esta área de investigación que combinan la metodología CCRT y el proceso de duelo (García Mantilla, López del Hoyo, Ávila-Espada, y Pokorny, 2011; García Mantilla, 2012).

\section{Método}

\section{Participante}

Este es un estudio de caso único. A la paciente se le ha dado el seudónimo de Aída por motivos de confidencialidad. Es una mujer latina de 30 años de edad que se dedica al magisterio. En diferentes ocasiones solicitó un espacio psicoterapéutico refiriendo que el motivo de consulta eran los constantes problemas conyugales. En esta última ocasión, Aída solicitó psicoterapia debido a que el marido, hacia un mes había fallecido repentinamente en un accidente automovilístico. Refiriendo que se sentía enojada por dicho suceso.

La relación de pareja empezó en la juventud. Estuvieron 14 años juntos, de los cuales diez años fueron de noviazgo y cuatro años casados. Al relatar sobre la relación que tenían comenzó diciendo que al principio pasaban momentos agradables, pero que desde el inicio existieron agresiones verbales por parte de él que ella permitió. Al paso del tiempo los ataques verbales fueron acentuándose y se sentía poco valorada, querida e incluso insignificante si se comparaba con él. Relata que a consecuencia de la mala relación ambos fueron infieles, pero su pareja debido a éste evento la martirizaba constantemente, por lo que la relación se tornó más 
conflictiva.

Al explorar su funcionamiento en la infancia mencionó que había sido insegura, le costaba trabajo defenderse y su manera de reaccionar frente a las críticas familiares era el llanto. Aída es la segunda de cinco hijos y relata que aunque el padre le impulsó a que estudiara, ella no se arriesgó a estudiar diferentes cosas por miedo a fracasar y se describió como una persona conformista. Relató que en la relación de sus padres hubo maltrato. Aceptó que ese era el caso de su relación y aunque ella había contribuido a ello, el mayor culpable de que la relación se tornara destructiva era la pareja, ya que éste ignoraba por completo sus emociones y ella dejó de pedirle que le prestara atención y empezó a buscar en otro lado lo que sentía que le hacía falta.

La muerte repentina del esposo fue lo que la llevó a pedir consulta ya que se encontraba experimentando una serie de emociones encontradas. Se trabajó con una modalidad de psicoterapia psicodinámicas de tiempo limitado, focalizando en trabajar la frustración y enojo exacerbados hacia el esposo a raíz de su muerte, comprendiendo la dinámica de la relación ambivalente (amor-odio) y dependiente, para poder facilitar la expresión de sentimientos entremezclados por el impacto de la pérdida súbita, la cual imposibilitó el perdón entre ambos. Teniendo en cuenta como foco complementario el trabajo de la ansiedad de separación por el fin de la terapia.

\section{Instrumento}

El CCRT, fue desarrollado por Lester Luborsky y Paul Crits-Christoph (1976, 1990, 1998) en la Universidad de Pennsylvania (EUA). El CCRT, es el patrón que cada persona sigue al relacionarse con los demás y se deriva a través de la consistencia que se encuentra en las narraciones que las personas cuentan sobre sus relaciones"(Luborsky, 1998b). Numerosos estudios han demostrado pruebas sobre la validez y buena fiabilidad del método en sus tres componentes ( $\mathrm{D}: \mathrm{k}=60$; RO:k=.68; RS:k=.71), (Luborsky y Crits-Christoph, 1990, 1998; Luborsky et al., 1999), pero con frecuencia otras discusiones han incluido críticas sobre la estructura de categorías (Albani et al., 2002; Strauss et al., 1995), dando como resultado la reformulación alemana.

Mediante el método se trata de identificar el tema central de conflicto. De este modo, cuantos más episodios de relación se obtengan del material clínico, más precisa será dicha representación. A estas narraciones se les aplica dos fases de evaluación con el método CCRT: La Fase A, consiste en localizar e identificar los episodios de relación y la Fase B, consiste en extraer el CCRT de un set de narraciones. A continuación se explican de manera general el procedimiento (para más detalle consultar: A guide to the CCRT method, Luborsky, 1998).

En la fase A se seleccionan los episodios de relación y se debe marcar el objeto; el tiempo en y la puntuación. La fase B se compone de dos pasos: el primero consiste en que el juez identifique los tipos de componentes que serán evaluados en cada uno 
de los episodios de relación; y el segundo requiere que el juez sume el total de estos componentes para crear el CCRT. Cada uno de los componentes es codificado a partir de una misma lista de predicados de estructura lógica organizada jerárquicamente. Tanto los Deseos como las Respuestas se ajustan a la misma lista de categorías. La subdivisión de si la función recae en el sujeto o en el objeto también es diferente a la del método tradicional; es decir que los deseos y las respuestas se dividen en ocho componentes en vez de cuatro [véase Tabla 1, (Albani et al., 2002)].

Tabla 1. Componentes del CCRT-LU

\begin{tabular}{|c|c|c|c|c|c|c|c|}
\hline \multicolumn{4}{|c|}{ DESEOS } & \multicolumn{4}{|c|}{ RESPUESTAS } \\
\hline \multicolumn{2}{|c|}{$\begin{array}{l}\text { Deseo del otro } \\
\text { (DO) }\end{array}$} & \multicolumn{2}{|c|}{$\begin{array}{c}\text { Deseo del sujeto } \\
\text { (DS) }\end{array}$} & \multicolumn{2}{|c|}{$\begin{array}{c}\text { Respuesta del otro } \\
\text { (RO) }\end{array}$} & \multicolumn{2}{|c|}{$\begin{array}{l}\text { Respuesta del sujeto } \\
\text { (RS) }\end{array}$} \\
\hline $\begin{array}{l}\text { DOO } \\
\text { El otro } \\
\text { debería } \\
\text { hacerse a } \\
\text { sí mismo } \\
\text { o a otro }\end{array}$ & $\begin{array}{l}\text { DOS } \\
\text { El otro } \\
\text { debería } \\
\text { hacerme } \\
\text { a mí }\end{array}$ & $\begin{array}{l}\text { DSO } \\
\text { Yo quiero } \\
\text { hacer } \\
\text { al otro }\end{array}$ & $\begin{array}{l}\text { DSS } \\
\text { Yo quiero } \\
\text { hacerme } \\
\text { a mí } \\
\text { mismo }\end{array}$ & $\begin{array}{l}\text { ROO } \\
\text { El otro } \\
\text { hace a } \\
\text { sí mismo } \\
\text { o a otro }\end{array}$ & $\begin{array}{l}\text { ROS } \\
\text { El otro } \\
\text { me hace } \\
\text { a mí }\end{array}$ & $\begin{array}{l}\text { RSO } \\
\text { Yo hago } \\
\text { al otro }\end{array}$ & $\begin{array}{l}\text { RSS } \\
\text { Yo me } \\
\text { hago a } \\
\text { mí mismo }\end{array}$ \\
\hline
\end{tabular}

Nota. Las categorías DOO y ROO son de menor importancia para el CCRT.

La capacitación y calibración en el uso del CCRT-LU-S se llevó a cabo en la Universidad de Salamanca con un investigador de la misma con fiabilidad probada como supervisor del método. Los jueces que participaron en esta investigación fueron dos investigadores de doctorado con orientación psicodinámica. El primer juez estuvo encargado de extraer los episodios de relación (ER), tomando en cuenta las siguientes variables: 1) localizar el inicio y final del episodio, 2) el objeto de interacción y 3) la completud del episodio en una escala del 1 al 5; este procedimiento se basó en lo propuesto por Luborsky y Diguer (1998). El segundo juez acotó todos los componentes CCRT que serían puntuados y calificó cada uno de los componentes utilizando el sistema de categorías CCRT-LU-S.

Esta versión reformulada del CCRT-LU cuenta con 13 clusters, que son las categorías de alto nivel; 30 categorías de nivel medio, 119 subcategorías y las dos clases de armonioso y no armonioso. La evaluación de las categorías "hechas a medida" se realiza comenzando por las categorías de alto nivel o cluster, después se asigna una categoría de nivel medio y por último se ajusta a una categoría de nivel inferior o subcategorías; este tipo de evaluación es denominado de arriba-abajo. Para mayor entendimiento del método se anexa el siguiente cuadro que incluye los 13 clusters (véase Cuadro 1). 
Cuadro 1. Categorías de alto nivel del CCRT-LU

\begin{tabular}{ll}
\hline A. & Atender a \\
B. & Apoyar \\
C. & Amar, sentirse bien \\
D. & Tener autodeterminación \\
E. & Deprimirse, resignarse \\
F. & Estar insatisfecho, asustado \\
G. & Estar determinado por otros \\
H. & Estar enfadado, ser antipático \\
I. & Ser poco fiable \\
J. & Rechazar \\
K. & Dominar \\
L. & Molestar, atacar \\
M. & Retirarse en uno mismo \\
\hline
\end{tabular}

\section{Procedimiento}

El caso que se presenta fue trabajado en un centro de atención psicopedagógico perteneciente a una Universidad privada. La selección de la paciente se hizo conforme a los criterios del protocolo de investigación del diplomado en psicoterapia breve (100hrs) en que la psicoterapeuta se formó. El coordinador de la clínica donde se realizaron las prácticas del diplomado realizó la primera entrevista a la paciente que solicitó el servicio de atención psicológica para detectar si era candidata a esta modalidad de intervención. El terapeuta trabajó con un modelo focal y un modelo breve, combinando aspectos de Balint, Malan y Levenson. En esta intervención combinada, el encuadre propuesto fue delimitado a 15 sesiones, en donde las dos primeras sesiones se dedicaron a la entrevista y se planificó un seguimiento al mes. La frecuencia de sesiones fue de una vez por semana con duración promedio de 50 minutos cada una. Las sesiones fueron vídeograbadas, con consentimiento expreso de la paciente y con las garantías de confidencialidad necesarias. El terapeuta que trabajó con la paciente es psicóloga clínica, especializada en psicoterapia psicodinámicas de tiempo limitado. El caso estuvo sujeto sistemáticamente a supervisión por parte de un psicoterapeuta psicoanalítico, docto en psicoterapias psicodinámicas de tiempo limitado y especialista en procesos psicoterapéuticos.

El material video grabado de las sesiones fue transcrito por estudiantes de psicología. La reproducción de cada una de las sesiones derivó un informe utilizando el formato propuesto por (Balint, Ornstein y Balint, 1985), el cual actuó como base de los registros del proceso terapéutico y brindó organización al material. La base de donde se extrajeron los datos fue la transcripción de las sesiones. Para poner a prueba la hipótesis de que el método CCRT se mostraría sensible en detectar el proceso de duelo y que los logros clínicos se reflejarían igualmente en cambios estructurales en el patrón conflictivo relacional central, se evaluaron y compararon las primeras cuatro sesiones (fase inicial) y las últimas cuatro sesiones (fase final), dando como resultado un total de ocho sesiones analizadas. De las ocho sesiones se 
obtuvieron un total de 84 episodios de relación. En la fase inicial se obtienen un subtotal de 45 ER y en la fase final un subtotal de 39 ER. En cuanto a los episodios de relación sobre duelo obtenemos de ambas fases un total de 23 ER. En la fase inicial encontramos 9 ER y en la parte final14 ER.

\section{Análisis de los datos}

Este es un estudio de proceso a un caso único. La investigación cuenta con un objetivo clínico que es contribuir con la elaboración de duelo, y de manera independiente a la terapia se planteó como hipótesis que el método CCRT-LU-S se mostraría sensible en detectar el proceso de duelo y que los logros clínicos se reflejarían igualmente en cambios estructurales en el patrón conflictivo relacional central al comparar las fases inicial y final del tratamiento. El análisis de los datos se realizó a partir de las narraciones que se extrajeron del material clínico de la paciente. Estos episodios fueron evaluados con las dos fases del método. En la Fase A se identificaron los episodios de relación y en la Fase B, se extrajo el CCRT del set de narraciones.

En esta investigación prestaremos especial interés a los siguientes apartados extraídos de Fase B, los cuales tienen que ver con: a) el CCRT que se encontró en la fase inicial, final y global del tratamiento, b) los cambios en categorías con diferencias significativas al comparar el inicio y final de la terapia, y c) el análisis del CCRT de los episodios en donde se detectó el proceso de duelo en la fase inicial $\mathrm{y}$ final.

\section{a) Formulación y análisis del CCRT entre la fase inicial y la fase final de la terapia}

Para formular el CCRT se identifican los tipos de componente, es decir, los Deseos y las Respuestas. Se encontraron un total de 596 componentes en las ocho sesiones analizadas de la psicoterapia. Las respuestas ocupan la mayor cantidad con un total de 506 y los deseos la menor cantidad con total de 90 . Con el total de estos componentes se obtiene el CCRT de la paciente. Luborsky (1998a) refiere que la formulación final se compondrá de los Deseos y Respuestas que hayan aparecido con más frecuencia en los diferentes episodios de relación de cada sesión.

Por un lado, las primeras cuatros sesiones (fase inicial) dan como resultados la siguiente Formulación del CCRT: Deseo que el otro me ame, se sienta bien conmigo, yo deseo atenderlo y tener autodeterminación; el otro me molesta y ataca, yo lo atiendo y me siento insatisfecha y asustada. Por otro, lado las cuatro últimas sesiones (fase final) proporcionan la siguiente Formulación del CCRT: Deseo que el otro me apoye, yo amarlo y sentirme bien con el objeto y tener autodeterminación; el otro me molesta y ataca, yo le apoyo y me enfado con él pero tengo autodeterminación.

Al comparar las formulaciones de ambas fases vemos algunos cambios tanto en los Deseos como en las Respuestas. En cuanto a los Deseos, en la fase inicial la 
paciente desea que el otro (DOS) la ame, pero al final ese deseo cambia por apoyo. En un principio ella desea atender al otro (DSO) y al final lo que desea es ser amada. En cuanto a las Respuestas, al principio responde atendiendo a los demás (RSO), y al final existen con la misma frecuencia dos respuestas hacia el otro, por un lado da su apoyo pero también se siente molesta. Con respecto a su reacción hacia ella misma (RSS), al principio está asustada e insatisfecha, pero al final responde con más determinación (véase Tabla 2).

Tabla 2. Cambios del CCRT en la Fase Inicial y Final

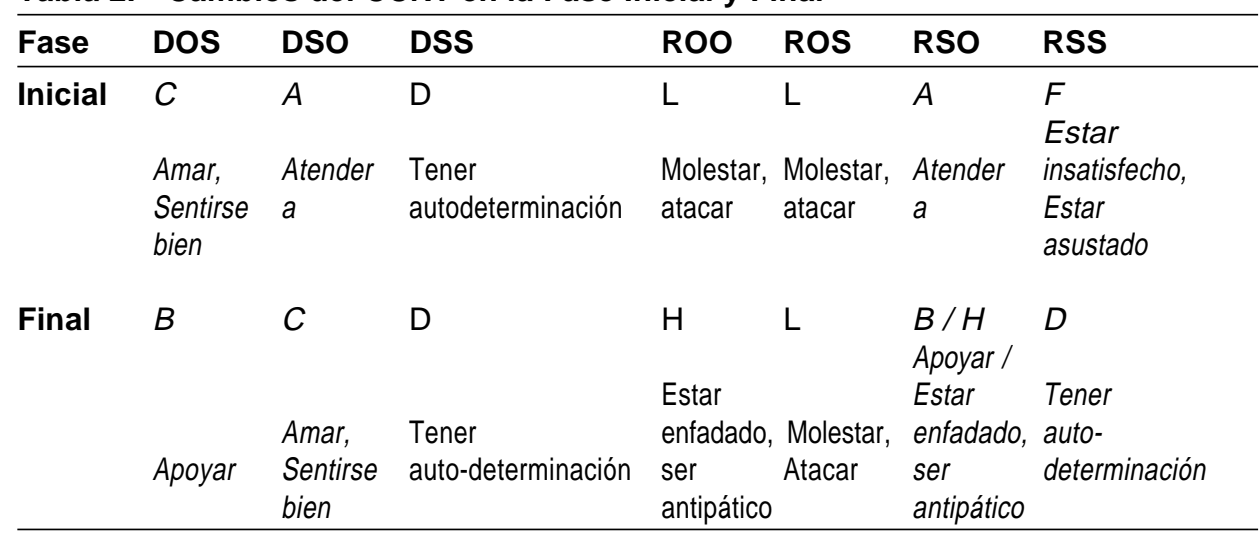

$\mathrm{Al}$ juntar las frecuencias de las categorías en las ocho sesiones, se extrae la siguiente Formulación global del CCRT: Deseo que el otro me apoye, me ame y se sienta bien conmigo; yo quiero amarlo y sentirme bien con él y deseo tener autodeterminación. El otro me ataca, yo le apoyo y estoy enfadada con él y me siento insatisfecha y asustada.

$\mathrm{Al}$ analizar la formulación global se puede decir que el conflicto existe entre lo que desea recibir del otro (DOS) y las respuestas que recibe (ROS); es decir, ella quiere que los demás la apoyen y la quieran pero las acciones de los demás son de ataque y rechazo. Frente a situaciones de conflicto ella reacciona de manera ambivalente (RSO); por un lado atiende y apoya pero también se siente enojada. En el transcurso de las sesiones su deseo hacia sí misma (DSS) ha sido constante, tener autodeterminación; sin embargo se responde a sí misma (RSS) de manera ambigua, por un lado estando insatisfecha y asustada y por el otro siendo fuerte.

\section{b) Categorías con diferencias significativas}

En el análisis de las frecuencias entre inicio y final del tratamiento de los componentes de Deseo y Respuesta se encontraron cambios significativos solamente en las siguientes respuestas (ROS) y (RSS): 
Tabla 3.

Categorías con diferencias significativas al comparar el inicio y final de la terapia

\begin{tabular}{|c|c|}
\hline ROS & RSS \\
\hline $\begin{array}{c}\mathrm{L} \\
\text { Molestar, atacar } \\
{ }^{*} \mathrm{~L}(p=0.046) .\end{array}$ & $\begin{array}{c}C \\
\text { Amar, sentirse bien } \\
{ }^{*} C(p=0.004) \\
D \\
\text { Tener autodeterminación } \\
{ }^{*} D(p=0.001) \\
G \\
\text { Estar determinado por otros } \\
{ }^{*} G(p=0.047) \\
H \\
\text { Estar enfadado, ser antipático } \\
{ }^{*} \mathrm{H}(p=0.033)\end{array}$ \\
\hline
\end{tabular}

* Se realizó el Test de Fisher para cada una de las categorías

En cuanto a la Respuesta del Objeto hacia el Sujeto (ROS), se mostró en ambas fases la categoría significativa de, L. Molestar, atacar, la cual redujo del $48 \%$ al 27\%, (véase Cuadro 1, Tabla 3 y Figura 1). Esta categoría refleja la mala relación que la paciente tenía con el marido pero también con otras figuras importantes de su vida como su padre. A raíz de la muerte del marido, la paciente se ha relacionado con la familia política y se suscitaron por el tipo de deceso de la pareja situaciones legales que complicaron la relación; sin embargo, al parecer Aída ha logrado establecer límites con la familia.

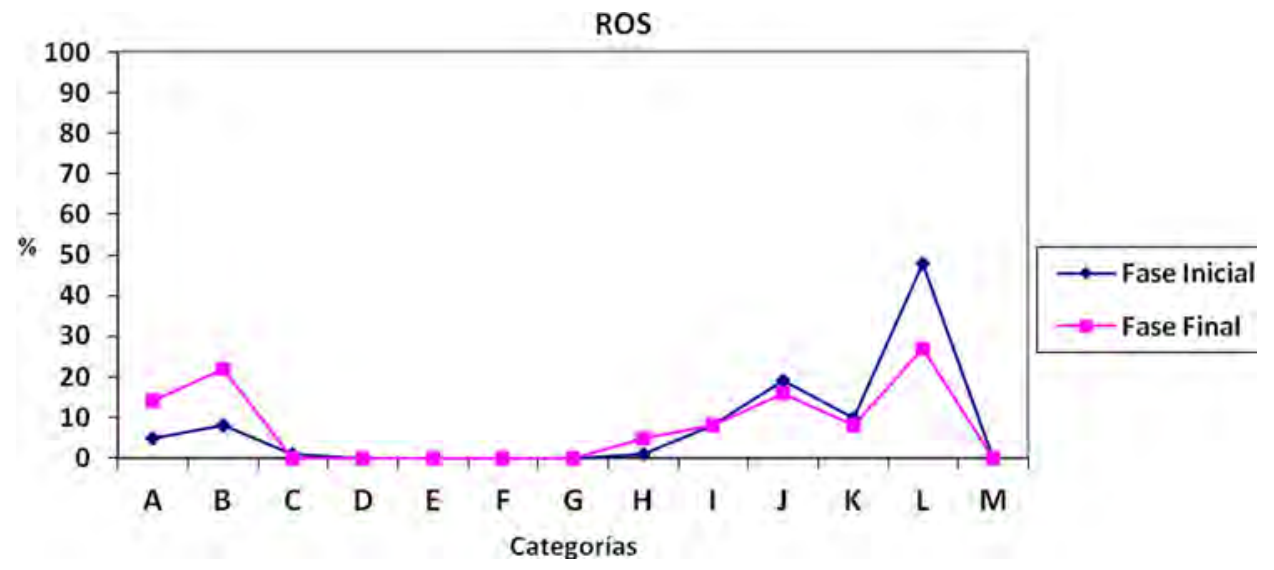

Figura 1. Diferencia en el componente ROS entre el principio y el final de la terapia

En cuanto a la Respuesta del Sujeto hacia sí mismo (RSS), se observó que al inicio de la terapia la categoría $F$. Estar insatisfecho, estar asustado, presentó el $28 \%$ y al final de la terapia fue la categoría $D$. Tener autodeterminación, la que 
incrementó significativamente del 3\% al 23\%. Del mismo modo, se observaron en este componente otros cambios significativos, por ejemplo, la categoría C. Amar, sentirse bien, incrementó del $4 \%$ al $17 \%$, la categoría G. Estar determinado por otros, decremento de $18 \%$ al $8 \%$ y la categoría $H$. Estar enfadado, ser antipático, disminuyó del $20 \%$ al 9\%, (véase Cuadro 1, Tabla 3 y Figura 2). Evidentemente las respuestas de miedo están relacionadas con el evento reciente al que se está enfrentando, el cual es un duelo de tipo súbito o de urgencia por la muerte de la pareja. A pesar de que desea superar las situaciones dolorosas a las que se ha enfrentado y ahora la dolorosa pérdida del esposo, es de esperarse que sus respuestas sean de temor ya que en un duelo de esta magnitud las consecuencias psicológicas se complican debido a la imprevisibilidad del evento. Sin embargo, se observó el intento de modificar la percepción de sí misma valorando más sus cualidades que defectos y a pesar de los sentimientos intensos de tristeza, la paciente, por su edad, cuenta con la energía que le ayuda a recomenzar.

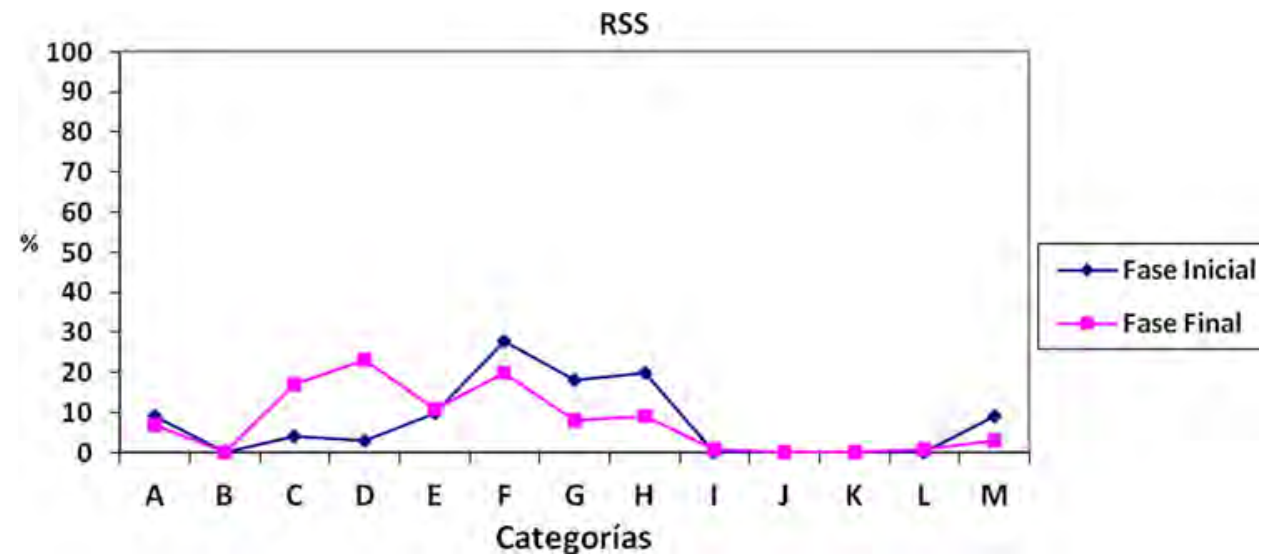

Figura 2. Diferencia en el componente RSS entre el principio y el final de la terapia

c) Análisis del CCRT de los episodios en donde se detectó el proceso de duelo

En este apartado se analizaron 23 ER sobre duelo, que se extrajeron del total de episodios del tratamiento. Debido a que el foco de trabajo fue la elaboración del duelo por la muerte del esposo, es de interés saber si el método identificó de manera sensible categorías que se refieran a dicho proceso.

El CCRT total que se extrajo de los episodios de duelo es el siguiente: Deseo que los otros me amen y se sientan bien conmigo, yo quiero amarlos y tener autodeterminación. El otro se ama a sí mismo y se retira en sí mismo, los demás me atacan, yo me enfado y me siento insatisfecha y asustada.

Ahora bien, al comparar el CCRT de la fase inicial y final del tratamiento, se observan cambios en tres de los ocho componentes, (DSO, ROO, ROS). Se analizan primero las categorías de alto nivel, posteriormente las de nivel medio y finalmente las subcategorías. 
En cuanto a las categorías de alto nivel (cluster), se observa lo siguiente, tanto para los Deseos como para las Respuestas: En cuanto a los deseos, la dimensión (DSO) al inicio presenta la categoría A. Atender $a$, y al final presenta la categoría C. Amar, sentirse bien.

En cuanto a las respuestas, se constata que en las categorías de alto nivel, el componente (ROO) al inicio destaca la categoría M. Retirarse en uno mismo y al final las categorías E. Deprimirse, resignarse; C. Amar, sentirse bien y M. Retirarse en uno mismo, que al final no cuenta con una categoría. En cuanto a la dimensión (ROS), al inicio resalta la categoría L. Molestar, atacar y al final las categorías, $I$. Ser poco fiable y L. Molestar, atacar.

Al hacer un análisis más profundo de las categorías de nivel medio, se encuentra lo siguiente en la fase inicial: por el lado de los deseos, el componente (DSO), cuenta con tres frecuencias C3. Confiar, estar satisfecho, sentir placer, D1. Ser interiormente fuerte y D2. Estar orgulloso, ser autónomo. Por el lado de las respuestas, la dimensión (ROO) cuenta con dos categorías, C3. Confiar, estar satisfecho, sentir placer y M3. Estar enfermo y el componente (RSS), tiene dos categorías H1. Sentir repulsa, estar enfadado y F2. Estar asustado, ansioso. En la fase final el componente (ROS) tiene las categorías I2. Ser egoista y L1. Molestar y la dimensión (RSS) cuenta con tres categorías E1. Estar desilusionado, F1. Sentirse culpable, avergonzado, estar insatisfecho y F2. Estar asustado, ansioso.

En cuanto a las subcategorías en la parte inicial por el lado de los deseos, el componente (DSS) cuenta con tres categorías frecuentes, C33. Expansionarse, ser espontáneo, desarrollarse libremente, ser feliz, sentirse bien, divertirse, pasarlo bien, D15. Soportar, resistir, aguantar, superar los obstáculos y D28. Cambiar desarrollar, mejorar. Por el lado de las respuestas la dimensión (ROO) cuenta con dos frecuencias C33. Expansionarse, ser espontáneo, desarrollarse libremente, ser feliz, sentirse bien, divertirse, pasarlo bien y M34. Morirse, suicidarse, el componente (RSS) adquiere tres frecuencias H16. Estar enfadado, montar cólera, sentirse frustrado por algo, E12. Estar desesperanzado, sufrir, estar afligido y F23. Estar nervioso, estar histérico, estar tenso, estar descontrolado. En la fase final la dimensión (DOS) obtiene dos frecuencias C11. Estar cerca, aceptar, ser íntimo, cuidar, ser bueno, ser cariñoso y C12. Consolar, reconfortar, en cuanto al componente (ROS) las frecuencias las ocupan I22. Ser deshonesto, ser injusto y L11.Hacer daño, herir, ofender, reñir, ridiculizar, denigrar, humillar y, por último, la dimensión (RSS) cuenta con cuatro categorías frecuentes, la categoría E12. Estar desesperanzado, sufrir, estar afligido, F11. Sentirse culpable, arrepentirse, F13. Sentirse mal, sentirse insatisfecho y por último F21. Estar ansioso, estar asustado, estar preocupado, evitar.

El CCRT de la fase inicial es el siguiente: Deseo que los otros me amen y se sientan bien conmigo, yo quiero atenderlos y tener autodeterminación. El otro se ama a sí mismo y se retira en sí mismo, los demás me molestan y atacan, yo me enfado con ellos y me siento insatisfecha y asustada. 
El CCRT de la fase final es el siguiente: Deseo que los otros me amen y se sientan bien conmigo, yo quiero amarlos y tener autodeterminación. Los demás son poco fiables, yo me molesto con ellos y me siento insatisfecha y asustada.

\section{Discusión general y conclusión}

En este caso se trabajó en la psicoterapia con la paciente la frustración y el enojo exacerbados hacia el esposo a raíz de su muerte; la relación entre ellos fue ambivalente. En el espacio psicoterapéutico se facilitó la expresión de sentimientos entremezclados por el impacto de la pérdida súbita, la cual dejó cosas inconclusas entre la pareja. Entre las metas se propuso que la paciente ganara independencia y elevara su autoestima, así como que lograra establecer límites claros en las relaciones. También se trabajó con el entendimiento de que tanto ella como el marido contribuyeron a generar la dinámica de una relación destructiva.

En este estudio de caso único, el proceso de duelo se puede complicar sin duda debido al tipo de perdida súbita, y a las variables que se involucran; por ejemplo, la relación ambivalente y dependiente con la pareja, la baja autoestima, la falta de confianza en sus relaciones con los demás, una familia distante, la indefensión aprendida y el tipo de muerte inesperada de una persona joven. Fue por ello de importancia una intervención y atención especializada debido a la imprevisibilidad y brusquedad del evento.

En la psicoterapia de tiempo limitado se propuso como foco dinámico, el facilitar el entendimiento de los sentimientos de enojo exacerbados a raíz de la muerte del esposo, a partir de trabajar con la relación autodestructiva y dependiente generada entre ambos, relación ambivalente (amor-odio), en donde él la maltrataba física y psicológicamente y ella se sentía con baja autoestima e incapaz de poner límites para defenderse, y a partir del entendimiento de esa dinámica de pareja se podría facilitar el perdón simbólico.

Ahora bien, el CCRT, se mostró sensible para detectar los procesos de cambio de manera significativa en algunas de las categorías, en donde se aplicó el Test de Fisher para medir el nivel de confianza. El método ofreció un medio para hacer operacionales los conflictos focales y centrales y detectó el foco de trabajo planteado a la paciente desde el inicio del proceso psicoterapéutico. Al comparar ambas fases del tratamiento se ven algunos cambios tanto en los deseos como en las respuestas. Aída, en la parte inicial, desea que los otros (DOS) la amen y se sientan bien con ella pero en la parte final el deseo que sobresale es el de ser apoyada. $\mathrm{Su}$ deseo hacia los demás (DSO), al inicio de la terapia es de estar al pendiente de los demás y al final de la terapia es de amar y sentirse bien con los demás. En cuanto a las respuestas de ella hacia los demás (RSO), al inicio se ve que responde atendiendo a las demandas pero al final sus respuestas son de apoyo pero igualmente de enfado por seguir siendo víctima de agresiones. Finalmente, al principio de las sesiones ella misma (RSS) se sentía temerosa y asustada, pero conforme transcurrió la terapia se sintió significativamente con más confianza y determinación a intentar 
salir adelante.

$\mathrm{Al}$ analizar la formulación final, el conflicto existe entre lo que desea recibir del otro (DOS) y las respuestas que recibe (ROS); es decir, ella quiere que los demás la apoyen y la quieran pero las reacciones de los demás hacia ella son de ataque y rechazo. Frente a situaciones de conflicto reacciona de manera ambivalente (RSO), por un lado atiende y apoya, pero eso mismo la hace sentirse molesta, ya que siente que los demás no le tienen consideración. En el transcurso de las sesiones su deseo hacia sí misma (DSS) ha sido constante, tener autodeterminación, y las respuestas que en ella predominan (RSS) son de insatisfacción y temor.

En cuanto al análisis del CCRT de los episodios en donde se detectó el proceso de duelo, se observaron cambios en tres componentes (DSO, ROO, ROS). Ella cambia su deseo de atender al los demás por el de amar y sentirse bien. Lo cual puede ser un indicador de que existe el deseo de cambiar su manera de relacionarse, sin embargo, las respuestas de los otros hacia ella siguen siendo negativas en donde perdura el ataque y surge el sentimiento de que los demás son poco confiables. Dichas respuestas de los otros pueden dificultar el proceso de elaboración del duelo. Al hacer un análisis más fino de las categorías se observa que éstas detectan de manera más sensible las emociones de la paciente en el proceso de duelo. Basándose clínicamente en el CCRT, se concluye que ella deseaba sentirse amada y apoyada por el marido, puesto que Aída constantemente le demostraba su cariño y arrepentimiento pero se sintió ofendida y rechazada por él, y aunque desea perdonar y ser fuerte no ha podido dejar de estar enojada tanto con él como consigo misma y se siente triste y descontrolada.

Los objetivos de la terapia se cumplieron en gran medida, aunque no de forma total. Aída comprendió que tenía que cuidarse de sí misma, enfrentando el reto de construir nuevos roles e interactuar con los demás aprendiendo a establecer límites. Definitivamente, el espacio terapéutico le brindó un espacio en donde pudo hablar sobre todas las emociones que le despertó esta pérdida intempestiva e igualmente le dio herramientas para continuar con su proceso de elaboración, cuya duración hasta el momento es indefinible.

Finalmente resaltamos que este trabajo hace una aportación al área de investigación que combinan la metodología CCRT y el proceso de duelo exponiendo el estudio de un caso único de pérdida de la pareja, donde se pretende hacer una investigación de proceso, abordando la especificidad de las estructuras emocionales, cognitivas e interpersonales que puedan movilizarse mediante estrategias de psicoterapia de tiempo limitado orientadas psicoanalíticamente, y comprobar, a través de la evaluación CCRT, si dichos cambios muestran una evolución saludable del proceso de duelo. 


\section{Referencias bibliográficas}

Albani, C., Pokorny, D., Blazer, G., Koenig, S., Geyer, M., Thomae, H. y Kächele, H. (2002). El analisis final de Amalie: Investigación de un proceso terapéutico psicoanalítico, según el modelo de proceso de Ulm, utilizando el método del Tema Central de Conflicto Relacional (CCRT). / The final analysis of AMALIE: A study of the psychoanalytic process with the Core Coflictual Relationsip Theme Mehod according to the Ulm Process Model. Intersubjetivo: Revista de Psicoterapia Psicoanalitica y Salud, 4, 45-46.

Anstadt, T., Merten, J., Ullrich, B. y Krause, R. (1996). Erinnern und Agieren. Zeitschrift für psychosomatische Medezin, 42, 34-55.

Balint, M., Ornstein, P.H. y Balint, E. (1985). La psicoterapia focal. Buenos Aires: Gedisa.

Bowlby, J. (1993). El apego y la pérdida: La pérdida, Volumen 3 (A. Báez, Trans.). Madrid: Espasa Libros (Original publicado en Inglés en 1980).

Brown, G. W. y Harris, T. (1978). The social origins of depression: A study of psychiatric disorder in women. Londres: Tavistock.

Freud, S. (1917). Duelo y Melancolía. En Obras Completas. (Vol. 14). Buenos Aires: Amorrortu.

García Mantilla, M.G., López del Hoyo, Y., Ávila Espada, A., y Pokorny, D. (2011). Evaluación del cambio mediante el método CCRT-LU-S en un caso de duelo no resuelto tratado mediante psicoterapia psicoanalítica focal. Revista Argentina de Clínica Psicológica XX, 5-13.

Garcia Mantilla M.G.(2012). El proceso de duelo en psicoterapia de tiempo limitado, evaluado mediante el método del Tema Centran de Conflicto Relacional (CCRT). (Tesis doctoral). Universidad Complutense de Madrid. Madrid: España

Grabhorn, R., Overbeck, G., Kernhof, K., Jordan, J. y Muller, T. (1994). Veränderung der Selbst-ObjektAbgrenzung einer ebgestörten Patientin im stationären Therapieverlauf. Psychotherapie, Psychosomatik, Medizinische Psychologie, 44, 273-283.

Grenyer, B., y Luborsky. L. (1998). Positive versus Negative CCRT Patterns. In: L. Luborsky, y P. Crits-Christoph (Eds). Understanding Transference: The Core Conflictual Relationship Theme method (pp. 55-63). Nueva York, NY: Basic Books.

Horowitz, M. J., Bonanno, G. A. y Holen, A. (1993). Pathological grief: Diagnosis and explanation. Psychosomatic Medicine, 55, 260-273.

Levenson, H. (1997). Guía práctica para una nueva terapia de tiempo limitado. Bilbao: Desclée De Brouwer. (Original publicado en Inglés en 1995)

López del Hoyo, Y., y Ávila, A. (2004). Manual interno de aplicación del método CCRT-LU-S. Manuscrito. Unidad de Investigación en Psicología Clínica y Psicoterapia. Universidad de Salamanca.

Luborsky, L. (1976). Helping alliances in psychotherapy: The ground work for a study of their relationship to its outcome. En J. Claghorn (Ed.), Successful psychotherapy (pp. 92-116). Nueva York: Brunner/Mazel.

Luborsky, L. (1977). Measuring a pervasive psychic structure in psychotherapy: The Core Conflictual Relationship Theme. En Freedman N, Grand S (Eds), Communicative Structures and Psychic Structures (pp.367-395). Nueva York, Plenum Press.

Luborsky, L. (1998a). A guide to the CCRT method. En L. Luborsky, y P. Crits-Christoph (Eds), Understanding Transference: The Core Conflictual Relationship Theme method (pp. 15-42). Nueva York, NY: Basic Books.

Luborsky, L. (1998b). The early life of the idea for the core conflictual Relationship theme method. En L. Luborsky, y P. Crits-Christoph (Eds). Understanding Transference: The Core Conflictual Relationship Theme method (pp. 3-13). New York, NY: Basic Books.

Luborsky, L., y Crits-Christoph, P. (1990). Understanding Transference: The Core Conflictual Relationship Theme method. Nueva York, NY: Basic Books.

Luborsky, L., y Crits-Christoph, P. (1998). Understanding transference: The Core Conflictual Relationship Theme method ( $2^{\text {nd }}$ ed.). Washington, DC, US: American Psychological Association.

Luborsky, L., Crits-Christoph, P., Friedman, S., Mark, D. y Schaffler, P. (1991). Freud transference template compared with the Core Conflictual Relationship Theme (CCRT). En M. Horowitz (Ed.), Person Schemas and Maladaptive Interpersonal patterns (pp.167-195). Chicago: University of Chicago Press.

Luborsky, L., Diguer, L., Kächele, H., Dahlbender, R., Waldinger, R., Freni, S., Krause, R., Frevert, G., Bucci, W., Drouin, M.-S., Fischmann, T., Seganti, A., Wischmann, T., Hori, S., Azzone, P., Pokorny, D., Staats, H., Grenyer, B., Soldz, S., Anstadt, T., Schauenburg, H., y Stigler, M. (1999). A guide to the CCRT's Methods, Discoveries and Future. http:www.sip.medizin.uni-ulm.de/Links/CCRT/ccrtframe.html.

Malan, D. H. (1963). La psicoterapia breve. Buenos Aires: Centro Editor de América Latina. 
Parkes, C.M. (1998). Bereavement: Studies of grief in adult life. Madison: International Universities Press.

Tizón, J.L. (2004). Pérdida, pena, duelo. Vivencias, investigación y asistencia. Madrid: Paidós.

Tizón, J.L., y Sforza, M. (2008). Días de duelo, encontrando salidas. Madrid, España: Alba.

Turvey, C.L., Carney, C., Arndt, S., Wallace, R.B., y Herzog, R. (1999). Conjugal loss and syndromal depression in a sample of elders aged 70 years or older. American Journal of Psychiatry, 156, 1596-1601.

Woof, W.R. y Carter, Y.H. (1997). The grieving adult and the general practitioner: A literature review in two parts. British Journal of General Practice, 47, 443-448 y 509-514.

Worden, J.W. (2004). El tratamiento del duelo: Asesoramiento psicológico y terapia. Barcelona: Paidós (Original publicado en 2002). 